\title{
RELATIONS BETWEEN HOLE VOLUME AND MACROSCOPIC VOLUME IN VARIOUS POLYMERS
}

\author{
G. Dlubi $i^{a, b} ;$ M.A. Alam ${ }^{b}$, K. SaARinen ${ }^{c}$, J. Stejny ${ }^{b}$ \\ AND H.M. FRETWELL ${ }^{b}$
}

aTTA Institut für Innovative Technologien GmbH, Köthen, Aussenstelle Halle Edvard-Grieg-Weg 8, 06124 Halle (S.), Germany

${ }^{b}$ H.H. Wills Physics Laboratory, University of Bristol

Tyndall Avenue, Bristol BS8 1TL, U.K.

${ }^{\mathrm{c}}$ Laboratory of Physics, Helsinki University of Technology

P.O. Box 1000, 02150 HUT, Finland

Different definitions of the free volumes are discussed and related to the total specific volume and to the local free (hole) volume. Positron lifetime measurements are reported for polyethylene, polytetrafluoroethylene, and CR39-copolymers. Two long-lived components appear in polyethylene and polytetrafluoroethylene, which were attributed to o-Ps annihilations in crystalline regions and in holes of the amorphous phase. From a relation between the coefficients of the thermal expansion of macroscopic and hole volume, the fractional hole volume $h$ and from this the number of holes, $N$, is estimated. Values of $T_{\mathrm{g}}=195 \mathrm{~K}, h_{\mathrm{g}}=4.5 \%$ (polyethylene) and $5.7 \%$ (polytetrafluoroethylene) and $N_{\mathrm{g}}=0.73 \mathrm{~nm}^{-3}$ (polyethylene) and $0.36 \mathrm{~nm}^{-3}$ (polytetrafluoroethylene) were obtained. In semicrystalline polymers these values agree with estimates obtained from the densities of the crystalline and amorphous phases. The effect of cross-linking on the free-volume properties of CR39-copolymer networks was studied. The comparison of the hole volume with the specific volume allowed us to estimate the number density of holes to $\approx 1 \mathrm{~nm}^{-3}$.

PACS numbers: $61.41 .+\mathrm{e}, 65.70 .+\mathrm{y}, 78.70 .-\mathrm{g}$

\section{Introduction}

Polymers contain cavities or holes of atomic and molecular dimension which arise because of irregular molecular packing in the amorphous phase. In this paper we analyse positron lifetime measurements, performed on polyethylene $(\mathrm{PE})$ and polytetrafluoroethylene (PTFE) in the temperature range $80 \mathrm{~K}$ to $300 \mathrm{~K}$, and assess the size of the local free volume. Moreover, a copolymer with decreasing density of cross-links is investigated. 


\section{Experimental}

Positron lifetime measurements were performed using a fast-fast coincidence device with a time resolution of $257 \mathrm{ps}$, a channel width of $54.9 \mathrm{ps}$, and a $10^{6} \mathrm{~Bq}$ ${ }^{22} \mathrm{Na}$ positron source. During each measurement $2 \times 10^{6}(\mathrm{PE}, \mathrm{PTFE})$ or $7 \times 10^{6}$ (CR39) total counts were collected. Following source and background corrections, the lifetime spectra were analysed with the help of the discrete-term program LIFSPECFIT and with MELT.

\section{Results and discussion}

The lifetime spectra in PE and PTFE show four components, the two long-lived ones are attributed to o-Ps annihilation in free volumes in the crystalline $\left(\tau_{3}=1.0-1.2 \mathrm{~ns}\right)$ and amorphous phases $\left(\tau_{4}=1.5-2.7 \mathrm{~ns}\right.$ in $\mathrm{PE}$ and $2.5-4.3 \mathrm{~ns}$ in PTFE) [1]. From the o-Ps lifetimes the hole volumes $v$ are estimated using the well-known formula developed by Tao, Eldrup, and Jean and collaborators [2]. The hole volume $v=v_{c}$, estimated from $\tau_{3}$ and attributed to crystals, shows a weak linear increase (Fig. 1) due to the thermal expansion of the interstitial free volume. The $v=v_{\text {a }}$ (estimated from $\tau_{4}$ ) vs. $T$ dependence of the amorphous phase shows two straight lines with slopes $\alpha_{\mathrm{h}, \mathrm{g}}=\left(1 / v_{\mathrm{g}}\right)\left(\mathrm{d} v_{\mathrm{a}} / \mathrm{d} T\right)_{\mathrm{g}}$ and $\alpha_{\mathrm{h}, \mathrm{r}}=\left(1 / v_{\mathrm{g}}\right)\left(\mathrm{d} v_{\mathrm{a}} / \mathrm{d} T\right)_{\mathrm{r}}$ where $v_{\mathrm{g}}=v_{\mathrm{a}}\left(T_{\mathrm{g}}\right)$. The lines cross at a temperature which may be interpreted as glass transition temperature. $T_{\mathrm{g}}=195 \mathrm{~K}$ was found for both PE and PTFE.

The free volume may be defined as the volume in an amorphous polymer being in excess to a hypothetical equilibrium volume [3], which we denote as bulk

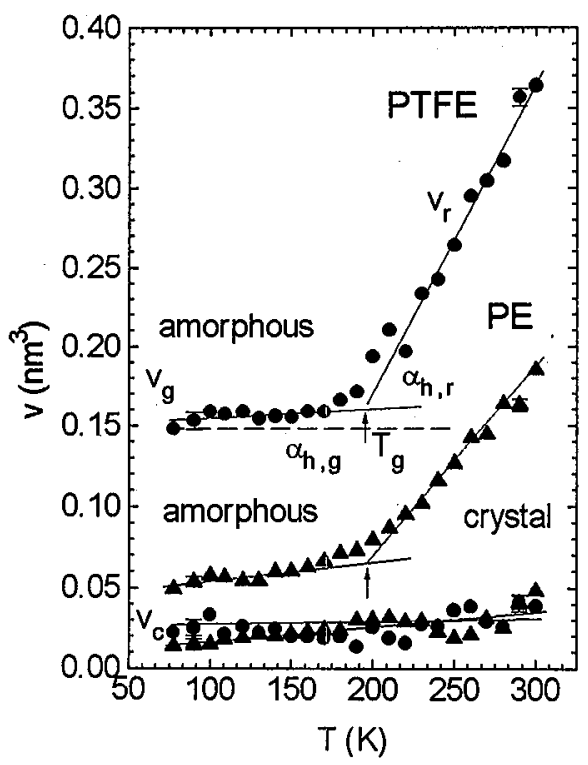

Fig. 1. Temperature dependence of the mean hole volume in amorphous $\left(v_{\mathfrak{g}}, v_{\mathrm{r}}\right)$ and crystalline $\left(v_{c}\right)$ phases of PE and PTFE. 
volume, $V_{\mathrm{b}}=V_{\mathrm{b}}(0)\left(1+\alpha_{\mathrm{b}} T\right)$. $\alpha$ denotes the (cubic) coefficient of thermal expansion of the volume of bulk $\left(\alpha_{\mathrm{b}}\right)$, glassy $\left(\alpha_{\mathrm{g}}, T<T_{\mathrm{g}}\right)$, rubbery $\left(\alpha_{\mathrm{r}}, T>T_{\mathrm{g}}\right)$, and crystalline $\left(\alpha_{c}\right)$ phases. In amorphous polymers, the bulk volume at absolute zero, $V_{b}(0)$, may be estimated from the linear extrapolation of total volume $V_{\mathrm{r}}=V_{\mathrm{b}}(0)\left(1+\alpha_{\mathrm{r}} T\right)$ from the rubbery phase down to $0 \mathrm{~K}$ [3]. Like the crystals, the bulk of the amorphous phase expands due to the anharmonicity of atomic oscillations. $\alpha_{b}$ is not known, but may be approximated by $\alpha_{\mathrm{g}}$. For semicrystalline polymers, the specific bulk volume may be approximated by the specific volume of the crystalline phase, $V_{\mathrm{b}}(0)=V_{\mathrm{c}}(0), \alpha_{\mathrm{b}}=\alpha_{\mathrm{c}}$ and $V_{\mathrm{b}}(T)=V_{\mathrm{c}}(T)$ [3]. It represents the most dense packing of a given polymer and is composed of the actually occupied volume - the van der Waals-volume $V_{\mathrm{W}}[3]$ - and the interstitial free volume, that is the supplement of the crystalline packing coefficient $K$ to $100 \%$. The fractional free volume $h$ (or $f$ ) at a given temperature may be estimated from

$$
h=\left(V_{\mathrm{a}}-V_{\mathrm{c}}\right) / V_{\mathrm{a}}=1-V_{\mathrm{c}} / V_{\mathrm{a}}=1-\rho_{\mathrm{a}} / \rho_{\mathrm{c}},
$$

where $V_{\mathrm{a}}=1 / \rho_{\mathrm{a}}$ and $V_{\mathrm{c}}=1 / \rho_{\mathrm{c}}$ denote the specific volume and $\rho_{\mathrm{a}}$ and $\rho_{\mathrm{c}}$ - the density of the polymer in the amorphous (either glassy or rubbery) phase and in the crystalline phase.

From empirical data it was found that $V_{\mathrm{b}}(0)=1.3 V_{\mathrm{W}}[3]$, the interstitial free volume amounts to $0.3 V_{\mathrm{W}}$. With this approximation and $\alpha_{\mathrm{b}}=\alpha_{\mathrm{g}}$ the fractional free volume is given by

$$
h=\left[V(T)-1.3 V_{\mathrm{W}}\left(1+\alpha_{\mathrm{g}} T\right)\right] / V(T) .
$$

The coefficient of thermal expansion of the total volume may be written as $\alpha=\alpha_{\mathrm{h}} h+\alpha_{\mathrm{b}}(1-h)$. From this $h=\left(\alpha-\alpha_{\mathrm{b}}\right) /\left(\alpha_{\mathrm{h}}-\alpha_{\mathrm{b}}\right)$ follows. Ignoring the thermal expansion of the bulk, $\alpha_{\mathrm{b}}=0$, one obtains $h=\alpha / \alpha_{\mathrm{h}}$. Assuming $\alpha_{\mathrm{b}}=\alpha_{\mathrm{c}}$ or $\alpha_{\mathrm{b}}=\alpha_{\mathrm{g}}$ we may calculate a more accurate value for $h$. Hristov et al. [4] assumed that the previous relation may be valid for both temperature ranges; $T<T_{\mathrm{g}}$ and $T>T_{\mathrm{g}}$. The value $\alpha_{\mathrm{b}}$ can be eliminated and one obtains for $T \rightarrow T_{\mathrm{g}}$

$$
h_{\mathrm{g}}=\left(\alpha_{\mathrm{r}}-\alpha_{\mathrm{g}}\right) /\left(\alpha_{\mathrm{h}, \mathrm{r}}-\alpha_{\mathrm{h}, \mathrm{g}}\right) \text {. }
$$

Here $\alpha_{\mathrm{r}}-\alpha_{\mathrm{g}}$ is the change of the coefficient of thermal expansion of the macroscopic volume at $T_{\mathrm{g}}, \alpha_{\mathrm{h}, \mathrm{r}}-\alpha_{\mathrm{h}, \mathrm{g}}$ denotes the same for the hole volume. The value of $h\left(T_{\mathrm{g}}\right)=h_{\mathrm{g}}$ may be estimated from Eq. (3) using $\alpha_{\mathrm{g}}=6 \times 10^{-4} \mathrm{~K}^{-1}(\mathrm{PE})$, $\alpha_{\mathrm{g}}=1.3 \times 10^{-4} \mathrm{~K}^{-1}(\mathrm{PTFE}), \alpha_{\mathrm{r}}=13.5 \times 10^{-4} \mathrm{~K}^{-1}(\mathrm{PE}), \alpha_{\mathrm{r}}=8.3 \times 10^{-4} \mathrm{~K}^{-1}$ $(\mathrm{PTFE})[3], \alpha_{\mathrm{h}, \mathrm{g}}=1.45 \times 10^{-3} \mathrm{~K}^{-1}(\mathrm{PE}), \alpha_{\mathrm{h}, \mathrm{g}}=0.13 \times 10^{-3} \mathrm{~K}^{-1}(\mathrm{PTFE}), \alpha_{\mathrm{h}, \mathrm{r}}=$ $18.1 \times 10^{-3} \mathrm{~K}^{-1}(\mathrm{PE})$, and $\alpha_{\mathrm{h}, \mathrm{r}}=12.4 \times 10^{-3} \mathrm{~K}^{-1}(\mathrm{PTFE})\left(T=T_{\mathrm{g}}=195 \mathrm{~K}\right.$, Fig. 1). We found $h_{\mathrm{g}}=4.5 \%$ (PE) and $h_{\mathrm{g}}=5.7 \%$ (PTFE).

We remark that $h_{\mathrm{g}}$ may be approximately estimated in the case that $\alpha_{\mathrm{r}}$ and $u_{\mathrm{g}}$ for a given polymer are not known. In this case we may use the Simha-Boyer value $\left(\alpha_{\mathrm{r}}-\alpha_{\mathrm{g}}\right) T_{\mathrm{g}} \approx 0.113$ (see [3]), and obtain

$$
h_{\mathrm{g}}=0.113 /\left[T_{\mathrm{g}}\left(\alpha_{\mathrm{h}, \mathrm{r}}-\alpha_{\mathrm{h}, \mathrm{g}}\right)\right] \text {. }
$$

With Eq. (4) $h_{\mathrm{g}}$ may be estimated using only positron data. We obtain $h_{\mathrm{g}}=3.5 \%$ (PE) and $h_{\mathrm{g}}=4.7 \%$ (PTFE) using $T_{\mathrm{g}}=195 \mathrm{~K}$. As a further approximation the relation $\left(\alpha_{r}-\alpha_{g}\right) \approx 5 \times 10^{-4} \mathrm{~K}^{-1}$ may be used [3] which, introduced in Eq. (3), leads to values of $h_{g}=3.0 \%$ (PE) and $h_{g}=4.1 \%$ (PTFE). 
From $h_{\mathrm{g}}=v_{\mathrm{g}} N_{\mathrm{g}}$ estimated from Eq. (3) we calculated the number density of holes at $T_{\mathrm{g}}$ to $N_{\mathrm{g}}=0.73 \mathrm{~nm}^{-3}$ for PE and $0.36 \mathrm{~nm}^{-3}$ for PTFE. With the known $N_{\mathrm{g}}$ we may now estimate the temperature dependence of fractional hole volume $h(T)$. From the relation $h(T)=N_{\mathrm{g}} v_{\mathrm{a}}(T)$ follows that $h$ should behave like $v_{\mathrm{a}}$ but weighted with $N_{\mathrm{g}}$. At this point we remark however that the hole volume $v_{\mathrm{a}}$ would expand also if $h=$ constant, that is for $\alpha=\alpha_{\mathrm{b}}=\alpha_{\mathrm{h}}$. Therefore we have to subtract this contribution from $N_{\mathrm{g}} v_{\mathrm{a}}(T)$. Doing this we obtain finally

$$
h=N_{\mathrm{g}}\left[v_{\mathrm{a}}(T)-v_{\mathrm{g} \alpha_{\mathrm{b}}}\left(T-T_{\mathrm{g}}\right)\right] .
$$

Since our reference point, $N_{\mathrm{g}} v_{\mathrm{g}}$, is at $T_{\mathrm{g}}$, Eq. (5) leads to a lowering (increasing) of $h$ compared with $N_{\mathrm{g}} v_{\mathrm{a}}(T)$ inthe temperature range $T>T_{\mathrm{g}}\left(T<T_{\mathrm{g}}\right)$. $\alpha_{\mathrm{b}}$ may be approximated by $\alpha_{\mathrm{g}}$. In the range $T<T_{\mathrm{g}}$ the slope in $h$ is distinctly lowered (PE) or possibly cancelled (PTFE). Actually, it is very often observed that the fractional free volume estimated from positron lifetime measurements and normalised at $T_{\mathrm{g}}$ with the help of $P V T$-data, agrees well with the fractional free volume estimated only from $P V T$-data, in the range $T>T_{\mathrm{g}}$, but is clearly smaller than these values below $T_{\mathrm{g}}$. This discrepancy may be removed, at least partially, by the correction term in Eq. (5).

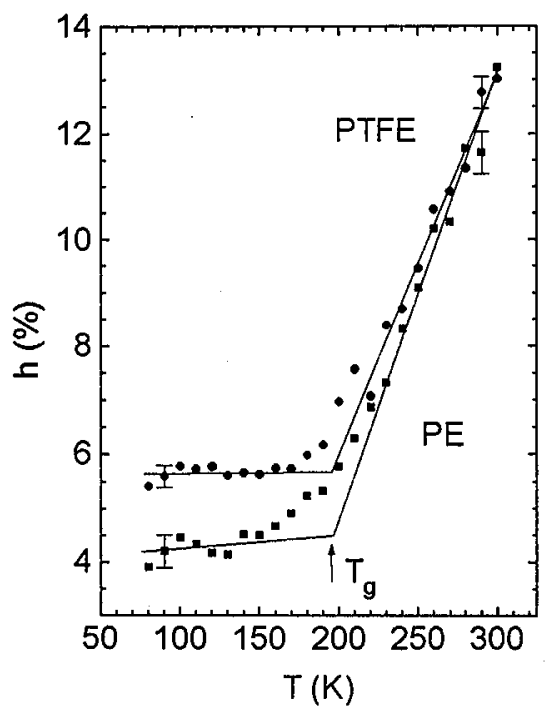

Fig. 2. Temperature dependence of the fractional free (hole) volume $h$ in the amorphous phase of PE and PTFE.

The results of our calculation using Eq. (5) are shown in Fig. 2. Between $80 \mathrm{~K}$ and $300 \mathrm{~K}$, the fractional free volume $h$ increases from $4.2 \%$ to $13.2 \%$ in $\mathrm{PE}$ and from $5.7 \%$ to $13.0 \%$ in PTFE. We may now compare these values with those obtained via Eq. (1). With $\rho_{\mathrm{a}}=0.85 \mathrm{~g} / \mathrm{cm}^{3}, \rho_{\mathrm{c}}=1 \mathrm{~g} / \mathrm{cm}^{3}(\mathrm{PE})$ and $\rho_{\mathrm{a}}=2.00 \mathrm{~g} / \mathrm{cm}^{3}, \rho_{\mathrm{c}}=2.35 \mathrm{~g} / \mathrm{cm}^{3}$ we obtain $h(300 \mathrm{~K})=15.0 \%(\mathrm{PE})$ and $14.9 \%$ (PTFE). With the hole volume $v_{\mathrm{a}}$ estimated from the $\tau_{4}$ lifetime at room temperature, $v_{\mathrm{a}}=0.188 \mathrm{~nm}^{3}$ and $0.372 \mathrm{~nm}^{3}$, we obtain $N=0.80 \times 10^{27} \mathrm{~m}^{-3}(\mathrm{PE})$ and 
$0.40 \times 10^{27} \mathrm{~m}^{-3}$ (PTFE). This values are in good agreement with the results estimated previously and may confirm the assumption $N(T)=N_{\mathrm{g}}=$ const. $h(300 \mathrm{~K})$ may be also estimated from Eq. (2). With $V=32.8 \mathrm{~cm}^{3} / \mathrm{mol}, V_{\mathrm{W}}=20.5 \mathrm{~cm}^{3} / \mathrm{mol}$ (PE), $V=50.0 \mathrm{~cm}^{3} / \mathrm{mol}, V_{\mathrm{W}}=32 \mathrm{~cm}^{3} / \mathrm{mol}(\mathrm{PTFE})$ and $\alpha_{\mathrm{b}}=\alpha_{\mathrm{g}}=0$, as it is very often assumed in the literature, we estimated $h(300 \mathrm{~K})=18.75 \%(\mathrm{PE})$ and $19.4 \%$ (PTFE). These results are clearly larger than those observed in Fig. 2. The reason is obviously that we had ignored the thermal expansion of the bulk volume. With $\alpha_{\mathrm{g}}=6 \times 10^{-4} \mathrm{~K}^{-1}(\mathrm{PE})$ and $\alpha_{\mathrm{g}}=1.3 \times 10^{-4} \mathrm{~K}^{-1}(\mathrm{PTFE})$ [3] we obtain $h(300 \mathrm{~K})=4.13 \%(\mathrm{PE})$ and $16.25 \%$ (PTFE). The value estimated for PE is clearly too low. This might be attributed to a too high $\alpha_{\mathrm{g}}$ value used for the semicrystalline PE, or to the approximation $\alpha_{\mathrm{b}}=\alpha_{\mathrm{g}}$. Using a lower $\alpha_{\mathrm{g}}=2.87 \times 10^{-4} \mathrm{~K}^{-1}$ found in Ref. [5] a more realistic value of $h(300 \mathrm{~K})=11.8 \%$ is estimated.

Ploy (diethylene glycol bis (allyl carbonate)) networks with a predetermined density of crosslinks were prepared by a bulk copolymerization of diethylene glycol bis (allyl carbonate), commonly known as CR-39 monomer, with different amounts of allyl ethoxyethyl carbonate [6]. Three lifetimes were observed, the long-lived component varied between $\tau_{3}=1.640 \mathrm{~ns}$ and $2.145( \pm 0.012) \mathrm{ns}$. In Fig. 3 we have plotted the dependence of the mean hole volume $v$ and of the specific volume $V$ (the inverse of the density) on the concentration $c$ of the monoallyl comonomer. On the top axis the glass transition temperature $T_{\mathrm{g}}$ corresponding to $c$ is also shown. For analysing the relations between $V$ and $v$, we assume that the change in the specific volume $V(c)$ with increasing concentration $c$ of monoallyl comonomer is controlled exclusively by the change in the total hole volume, $V(c)=V_{\mathrm{h}}(c)+V_{\mathrm{b}}(0)$.

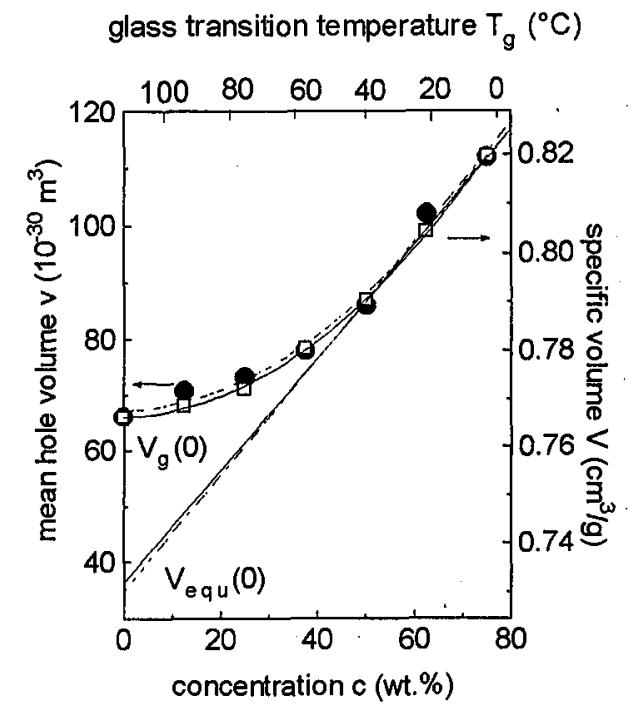

Fig. 3. Dependence of the mean hole volume $v$ (filled symbols, left axis) and of the macroscopic specific volume $V$ (empty symbols, right axis) on the concentration $c$ of the monoallyl comonomer (see text). On the top axis the glass transition temperature $T_{\mathrm{g}}$ is given which is related to $c$ of the bottom axis via $T_{\mathfrak{g}}\left[{ }^{\circ} \mathrm{C}\right]=111-1.428 c$ [wt.\%]. 
The total hole volume per gram is given by $V_{\mathrm{h}}(c)=N^{\prime}(c) v(c)$ where $N^{\prime}$ and $v$ denote the number of holes per gram and the mean hole volume. For $c=0$ we have $V_{\mathrm{h}}(0)=N^{\prime}(0) v(0)$ and $V_{\mathrm{b}}(0)=V(0)-N^{\prime}(0) v(0)$. With $V_{\mathrm{b}}(c)=V_{\mathrm{b}}(0)$

$$
V(c)=N^{\prime}(c) v(c)+\left[V(0)-N^{\prime}(0) v(0)\right]
$$

follows. In order to take into account a possible variation of $N^{\prime}$ with $c$ we assume that $N^{\prime}(c)$ changes as

$$
N^{\prime}=N^{\prime}(0)(1+A c) \text {. }
$$

Equations (6) and (7) may be now fitted to the experimental points using $N^{\prime}(0)$ and $A$ as fitting parameters. From a least-squares fitting procedure we got $N^{\prime}(0)=(1 \pm 0.2) \times 10^{21} \mathrm{~g}^{-1}$ (corresponding to $1.3 \pm 0.3 \mathrm{~nm}^{-3}$ ) and $A=0.1 \pm 0.1$. Using $N^{\prime}(0)=1 \times 10^{21} \mathrm{~g}^{-1}, v(0)=0.066 \mathrm{~nm}^{3}$ and $V(0)=0.766 \mathrm{~cm}^{3} / \mathrm{g}$ we find $V_{\mathrm{b}}(c=0)=V(0)-N^{\prime}(0) v(0)=(0.700 \pm 0.013) \mathrm{cm}^{3} / \mathrm{g}$, a value only slightly larger to that estimated for the pure CR-39 from $V_{\mathrm{b}}(0)=1.3 V_{\mathrm{W}}=0.682 \mathrm{~cm}^{3} / \mathrm{g}$. Since Eq. (6) ignores the thermal expansion of the bulk volume $V_{b}$, the above estimation of $N=1.3 \pm 0.3 \mathrm{~nm}^{-3}$ may be considered as an upper limit of the true number density of holes. Further estimations [6] showed that a number of $N=$ $(1.0 \pm 0.4) \times 10^{27}$ holes per $\mathrm{m}^{3}$ may be accepted as a realistic value.

\section{References}

[1] G. Dlubek, K. Saarinen, H.M. Fretwell, J. Polymer Sci. B, Polym. Phys. 36, 1513 (1998); Nucl. Instrum. Methods Phys. Res. B 142, 139 (1998).

[2] Y.C. Jean, Mater. Sci. Forum 175-178, 59 (1995).

[3] D.W. Van Krevelen, Properties of Polymers, Elsevier, Amsterdam 1990.

[4] H.A. Hristov, B. Bolan, A.F. Ye, L. Xie, D.W. Gidley, Macromolecules 29, 8507 (1996).

[5] A.M. Aharoni, J. Polymer. Sci., Symposium 42, 795 (1973).

[6] G. Dlubek, J. Stejny, M.A. Alam, Macromolecules 31, 4574 (1998). 